\title{
Diversity in Nucleotide Binding Site-Leucine-Rich Repeat Genes in Cereals
}

\author{
Jianfa Bai, Lourdes A. Pennill, Jianchang Ning, Se Weon Lee, \\ Jegadeesan Ramalingam, Craig A. Webb, Bingyu Zhao, Qing Sun, \\ James C. Nelson, Jan E. Leach, and Scot H. Hulbert ${ }^{1}$ \\ Department of Plant Pathology, Kansas State University, Manhattan, Kansas 66506-5502, USA
}

\begin{abstract}
The diversity of the largest group of plant disease resistance genes, the nucleotide binding site-leucine-rich repeat (NBS-LRR) genes, was examined in cereals following polymerase chain reaction (PCR) cloning and database mining. NBS-LRR genes in rice are a large and diverse class with more than 600 genes, at least three to four times the complement of Arabidopsis. Most occur in small families containing one or a few cross-hybridizing members. Unlike in Arabidopsis and other dicots, the class of NBS-LRR genes coding for a Toll and mammalian interleukin-1 receptor (TIR) domain were not amplified during the evolution of the cereals. Genes coding for TIR domains are present in the rice genome, but have diverged from the NBS-LRR genes. Most cereal genes are similar in structure to the members of the non-TIR class of dicots, although many do not code for a coiled-coil domain in their amino termini. One unique class of cereal genes, with $\sim 50$ members, codes for proteins similar to the N-termini and NBS domains of resistance genes but does not code for LRR domains. The resistance gene repertoire of grasses has changed from that of dicots in their independent evolution since the two groups diverged. It is not clear whether this reflects a difference in downstream defense signaling pathways.
\end{abstract}

[Supplemental material is available online at www.genome.org. The sequence data from this study have been submitted to GenBank under accession nos. AF516886-AF516895.]

Plants use a variety of different types of disease-resistance genes to detect the presence of pathogens and induce defense responses. The largest class of these genes code for proteins with nucleotide binding site (NBS) and leucine-rich repeat (LRR) domains (Bent 1996; Hammond-Kosack and Jones 1997; Hulbert et al. 2001). The Col0 ecotype of Arabidopsis has been estimated to carry $\sim 150$ genes coding for NBS-LRR proteins, or more if genes coding for truncated versions of the protein are considered, and the rice genome was estimated to carry even more (Meyers et al. 2002). No function other than disease resistance has yet been assigned to this large class of genes.

NBS-LRR genes in plants are typically divided into two classes depending on whether they code for a TIR domain (having homology to the intracellular domain of the Drosophila Toll and mammalian interleukin-1 receptors in their $\mathrm{N}$-terminus). The TIR group genes are composed of an N-terminal TIR domain, a central NBS domain, and a Cterminal LRR region. This group of genes has been observed only in dicot plant species (Meyers et al. 1999; Pan et al. 2000a, Goff et al. 2002). The non-TIR group is sometimes referred to as the coiled-coil (CC) group because they typically have CC domains at their $\mathrm{N}$ termini.

The sequences of the central portion of NBS-LRR genes, including the NBS domain, have been used extensively to identify and to classify these genes. The popular use of this

'Corresponding author.

E-MAIL shulbrt@plantpath.ksu.edu; FAX (785) 532-5692.

Article and publication are at http://www.genome.org/cgi/doi/10.1101/ gr.454902. domain stems from a number of reasons: The NBS domain has some conserved amino acid motifs that assist in cloning these genes via PCR amplification and recognizing them in databases; the conserved motifs assist in aligning the sequences for phylogenetic analyses, and classification of NBS-LRR genes by their NBS region sequences accurately predicts whether they belong to the TIR or non-TIR class (Meyers et al. 1999; Pan et al. 2000a).

While LRR regions typically appear to be under strong diversifying selection pressure, NBS domains do not, or at least not to the same extent (Parniske et al. 1997; McDowell 1998; Meyers et al. 1998; Sun et al. 2001). This likely reflects the role of the LRR region in recognition of constantly evolving pathogen ligands and a role for the NBS domain in recognition signaling (Ellis et al. 1999; Ellis et al. 2000; Dodds et al. 2001). Analysis of the $L$ locus of flax has demonstrated that the TIR domain can also play a role in determining pathogen recognition and that it may be under diversifying selection like the LRR (Luck et al. 2000). LRR regions can also be difficult to use for comparative sequence analysis because even closely related genes often show size polymorphisms, making alignment difficult.

There are now sufficient sequences available from the cereals, especially rice, to reveal the diversity and general nature of NBS-LRR genes and related genes in cereal genomes. The present study was conducted to characterize the numbers and structures of NBS-LRR genes in rice for comparison with those of dicot species. Comparisons of these genes with those of other cereal species can be used to identify possible orthologs. We also describe a collection of probes that can be used for mapping and isolating these genes in different cereal crops. 
Bai et al.

\section{RESULTS}

\section{Isolation of NBS Clones for a Probe Collection}

Over 150 sequences were isolated by PCR amplification and cloning of the NBS region of rice and maize NBS-LRR genes using primers designed from conserved regions of known cereal NBS-LRR genes, or from sequences that were mined from the databases (see below). Most primers were designed to match the conserved P-loop motif marking the $\mathrm{N}$-terminal end of the NBS domain and a conserved MHD motif that occurs near the beginning of the LRR. This allowed isolation of the whole NBS region of the gene, corresponding to $\sim 900$ nucleotides. The majority of the clones were amplified from the rice cultivar Nipponbare. Nondegenerate primers designed from rice sometimes amplified maize DNA but usually worked poorly and provided only six unique clones from maize. Many primer pairs amplified several closely related genes. When these related gene fragments were used as probes in gel-blot hybridization experiments, they typically detected identical restriction fragments (data not shown). To make a collection of gene probes that would each identify different families of $\mathrm{R}$ genes (Table 1 ), only sequences that were sufficiently different from previously collected sequences were retained. An arbitrary cutoff of $75 \%$ amino acid identity was used to represent sequences from different families, because sequences with less identity than this typically identified different fragments in genomic hybridization experiments (data not shown). Using this criterion, the cloned rice probes were derived from 96 different NBS-LRR families.

\section{Collection of NBS Sequences by Database Mining}

The initial stages of NBS-LRR gene sequence collection consisted of mining sequences (mostly gene fragments) from GenBank and the Monsanto Rice Genome Sequence Database. Searches for rice sequences in the GenBank nonredundant and the high throughput genomic sequence databases were performed in March 2002 using a variety of predicted protein sequences from monocot and dicot NBS-LRR genes as queries. Examination of all sequences with TBLASTN scores $>1 \mathrm{e}-4$ yielded 216 rice bacterial artificial chromosomes (BACs) with one or more genes predicted to code for NBS-LRR proteins. An additional 61 gene fragments were identified, mostly from PCR-amplified genomic fragments including parts of NBS-coding sequences. The Monsanto database contained $259 \mathrm{Mb}$ of assembled sequence from the Japonica rice cultivar Nipponbare. We were able to collect 144 sequences that code for the complete NBS area, from the P-loop to the MHD motifs from this database. A very large Indica (line 9311) rice database ( $\mathrm{Yu}$ et al. 2002), consisting of assembled whole-genome shotgun sequences of $>360 \mathrm{Mb}$ of the rice genome, was also searched for NBS-LRR sequences. Approximately 560 different sequence contigs containing NBS-LRR sequences were identified in this database. Most of these contigs coded for at least one sequence of the complete NBScoding area of the gene (P-loop to MHD) and 253 predicted full-length genes were identified. Altogether, including sequences from the NBS-coding probe collection and the GenBank, Monsanto, and Indica databases, over 1080 sequences coding for complete NBS areas were examined. Pairwise comparisons of these sequences allowed the identification of identical sequences from different databases and their classification into different families. Genes were grouped into 354 different families, where members of a family share $>75 \%$ amino
Table 1. Predicted NBS-Coding Sequences Isolated From Rice by Cloning PCR Amplified Fragments

\begin{tabular}{|c|c|c|}
\hline $\begin{array}{l}\text { Clone } \\
\text { Name }\end{array}$ & Identical or Related Sequences* & Copy No.** \\
\hline rNBS1 & $\mathrm{AB} 017914$ & 1.1 \\
\hline rNBS2 & AP003275, IN35396 & 2.1 \\
\hline rNBS3 & IN7596 & 4.1 \\
\hline rNBS4 & IN2882 & 2.0 \\
\hline rNBS5 & IN15065, rNBS5b (88\%) & 0.8 \\
\hline rNBS6 & OSM148075 (97\%), IN1837 (97\%) & \\
\hline rNBS7 & OSM144390, IN19918 & \\
\hline rNBS8 & OSM151991 & 1.8 \\
\hline rNBS9 & OSM12017, IN2806 & \\
\hline rNBS10 & OSM1922 & 3.1 \\
\hline rNBS11 & IN4580 & 4.8 \\
\hline rNBS12 & OSM148064 & 2.0 \\
\hline rNBS13 & OSM120301, IN7733 & 1.0 \\
\hline rNBS14 & OSM133731 & 1.3 \\
\hline rNBS15 & OSM148062, AP003275a & 4.9 \\
\hline rNBS16 & OSM148567, IN15839 & 0.8 \\
\hline rNBS17 & OSM116395 & 1.0 \\
\hline rNBS18 & OSM1922, IN12788 & 1.5 \\
\hline rNBS19 & OSM122428, IN5476 & 3.9 \\
\hline rNBS20 & OSM1970 & 1.9 \\
\hline rNBS21 & OSM124828 & 1.0 \\
\hline rNBS22 & IN19, OSM129598, rNBS22b (87\%) & 2.0 \\
\hline rNBS23 & OSM133645, AP003839b, IN1044 & 1.0 \\
\hline rNBS24 & OSM138053, IN1277 & 1.4 \\
\hline rNBS25 & OSM144727, AP003995, IN16936 & 1.9 \\
\hline rNBS26 & OSM14020, IN34799 & 6.7 \\
\hline rNBS27 & OSM14023, AF032697 & 7.3 \\
\hline rNBS28 & IN20419 & 2.6 \\
\hline rNBS29 & OSM1916, AP3575b & 3.1 \\
\hline rNBS30 & OSM128453, IN637 & 3.6 \\
\hline rNBS31 & OSM11432, AP003914a, IN10063 & 3.3 \\
\hline rNBS32 & OSM11430, IN1053 & 4.3 \\
\hline rNBS33 & OSM117679 & 2.0 \\
\hline rNBS34 & OSM11429, AP003914d, IN7220 & 5.3 \\
\hline rNBS35 & OSM11430B, AP003914b & 2.4 \\
\hline rNBS36 & OSM13352 & 1.9 \\
\hline rNBS37 & OSM12017B, AF074894, IN12951 & 1.0 \\
\hline rNBS38 & OSM14018, AF032689 & 4.3 \\
\hline rNBS39 & OSM141732, IN14579 & 3.6 \\
\hline rNBS40 & OSM14676, IN23744 & 3.1 \\
\hline rNBS41 & OSM15374 & 3.1 \\
\hline rNBS42 & OSM19134 & 4.8 \\
\hline rNBS43 & OSM19134, IN459 & 5.3 \\
\hline rNBS44 & OSM135760 & 1.6 \\
\hline rNBS45 & IN1487 & 0.8 \\
\hline rNBS46 & OSM15354 & 3.9 \\
\hline rNBS48 & OSM11901, AC003621a, IN3280 & 2.1 \\
\hline rNBS49 & OSM113184, IN1169, rNBS49b (82\%) & 1.3 \\
\hline rNBS50 & OSM13480, AP003633, IN14668 & 2.4 \\
\hline rNBS52 & OSM15936, IN14071 & 1.5 \\
\hline rNBS53 & OSM15929, AF220740, IN17130 & 1.3 \\
\hline rNBS54 & OSM146, AP003840, IN8045 & 1.3 \\
\hline rNBS55 & OSM113712, IN13161 & 1.3 \\
\hline rNBS56 & OSM14162 & 2.9 \\
\hline rNBS57 & IN97289 & 1.1 \\
\hline rNBS58 & OSM142192, IN4 & 1.0 \\
\hline rNBS59 & ACO78890 & 2.3 \\
\hline rNBS60 & OSM150826, IN41105 & 8.2 \\
\hline rNBS62 & OSM14552, IN7190 & 0.6 \\
\hline rNBS63 & OSM146376, IN42153 & \\
\hline rNBS67 & OSM15999, AP003539d & \\
\hline rNBS68 & OSM11089, IN15589 & \\
\hline rNBS69 & OSM12066, IN130 & 1.0 \\
\hline rNBS70 & OSM140512 & 0.8 \\
\hline rNBS71 & AC083751, IN15208 & 3.0 \\
\hline rNBS72 & AC074354, IN3585 & 1.0 \\
\hline rNBS73 & OSM133123, IN41851 & 1.2 \\
\hline
\end{tabular}


Table 1. (Continued)

\begin{tabular}{|c|c|c|}
\hline $\begin{array}{l}\text { Clone } \\
\text { Name }\end{array}$ & Identical or Related Sequences* & Copy No.** \\
\hline rNBS74 & AL442107, IN48 & 1.3 \\
\hline rNBS75 & OSM15011, IN37552 & 1.9 \\
\hline rNBS76 & OSM12654, AP003799, IN3311 & 1.0 \\
\hline rNBS77 & OSM15610, AP003753, IN28943 & 1.8 \\
\hline rNBS78 & OSM13655 & 2.1 \\
\hline rNBS79 & OSM147688, IN13548 & 3.8 \\
\hline rNBS80 & OSM11170, IN26131 & 1.3 \\
\hline rNBS81 & OSM128851, IN10755 & 0.8 \\
\hline rNBS82 & OSM19131, AF220749, IN459 & 2.3 \\
\hline rNBS83 & AC079128b & \\
\hline rNBS84 & APO03269-2, IN-1507 & 3.0 \\
\hline rNBS85 & APO3219, IN112357 & 1.8 \\
\hline rNBS86 & AP003368, IN39058 & 1.5 \\
\hline rNBS87 & AL513003.1, IN541 & \\
\hline rNBS88 & IN41159 & \\
\hline rNBS89 & AP001168, IN8174 & \\
\hline rNBS90 & AP003073 (97\%), IN9666 (96\%) & \\
\hline rNBS92 & AP004223, АР003262 & \\
\hline rNBS95 & AP003616, IN16441 & \\
\hline rNBS96 & IN8880 & \\
\hline rNBS97 & OSM140690 & \\
\hline rNBS98 & AP003208, IN8376 & \\
\hline rNBS102 & AC090870, IN149 & \\
\hline rNBS104 & AP003563, IN1434 & \\
\hline rNBS106 & OSM14542, IN214 & \\
\hline rNBS108 & OSM150553 & \\
\hline rNBS109 & AC090870, IN22788 & \\
\hline rNBS111 & AP003609, IN5558 & \\
\hline rNBS112 & AC099402 & \\
\hline
\end{tabular}

*The most closely related sequences existing in GenBank (as of Jan. 2002), Monsanto, or Indica databases. Sequences from the Indica databases are designated by the prefix ' $I N$ ' and the sequence contig number. Sequences from the Monsanto database are designated 'OSM'. Values in parentheses give the approximate $\%$ sequence identity to the cloned sequence if $<98 \%$. ${ }^{* *}$ Copy No. is the average number of hybridizing bands when hybridized to gel blots of four rice cultivars digested with four different restriction enzymes (Fig. 1). Those where no number is shown were not tested.

acid sequence identity with other members. Efforts were made to isolate at least one full-length coding region for each family of NBS-LRR genes. These predicted coding regions can be searched or examined at (http://coding.plantpath.ksu.edu/ blast/blastNBS.html).

\section{Estimation of NBS-LRR Gene Number and Copy Number of Different Families}

Approximately 560 sequences predicted to code for NBS domains of NBS-LRR genes were identified in the Indica database. To project a total number of NBS-LRR genes in the rice genome, an estimate of the number of NBS-LRR genes missing from the Indica database was made. The predicted amino acid sequences of the 96 cloned NBS fragment probes were used in TBLASTN searches with the Indica sequences. The 96 sequences represent a diverse collection of genes (see below) and should represent an unbiased estimate of the coverage of the whole genome. Most of the sequences used in the search were from the Japonica cultivar Nipponbare, so that identification of Indica alleles for specific sequences was sometimes ambiguous. In most cases, near-perfect matches were identified; 74 of the 96 clones matched Indica sequences with $98 \%$ or better identity for at least 150 amino acids (Table 1 ). In other cases, presumed alleles were identified but sequence identity was lower. For example, two sequences, rNBS21 and rNBS70, which were estimated to exist in single copies in the genomes (below), were matched by single sequences in the Indica database that were $\sim 95 \%$ identical. If sequences with identities of $95 \%$ or better amino acid identity are considered probable alleles, then the Indica database carries alleles for all but 14 of the 96 sequences tested (85\%). If the 560 NBScoding sequences in this database represent $85 \%$ of the NBSLRR genes in the genome, the estimated number of genes would be $\sim 660$, which is very close to the estimate of Goff et al. (2002).

The average copy number of 73 of the rice NBS-region clones was estimated by hybridizing to DNAs of four rice varieties, each cut with four different enzymes (Fig. 1). The rice varieties examined included one Indica line (IR64), two Japonica types (Azucena and Gihobyeo) and cultivar Milyang23 derived from an Indica X Japonica cross. All of the probes hybridized to all four cultivars demonstrating that both rice subspecies generally carry the same families of NBS-LRR genes. The $\sim 30$ probes that appeared to detect single-copy genes typically revealed one or two bands in most enzyme digests of all four cultivars, although lanes in some digests were sometimes lacking bands, probably because small fragments migrated off the gel. Multiple-copy probes usually detected similar numbers of fragments in the different cultivars, with one exception. The rNBS41 probe identified an estimated five genes in the Azucena and Gihobyeo cultivars, but single genes in the other two. This copy number difference was also apparent when the sequence databases were examined. The Indica database carried a single gene that was highly similar to rNBS41, while the Monsanto database carried six sequences with $>70 \%$ amino acid identity. The probe therefore detects a family that has become amplified in some lines, possibly most Japonica types. The number of hybridizing re-

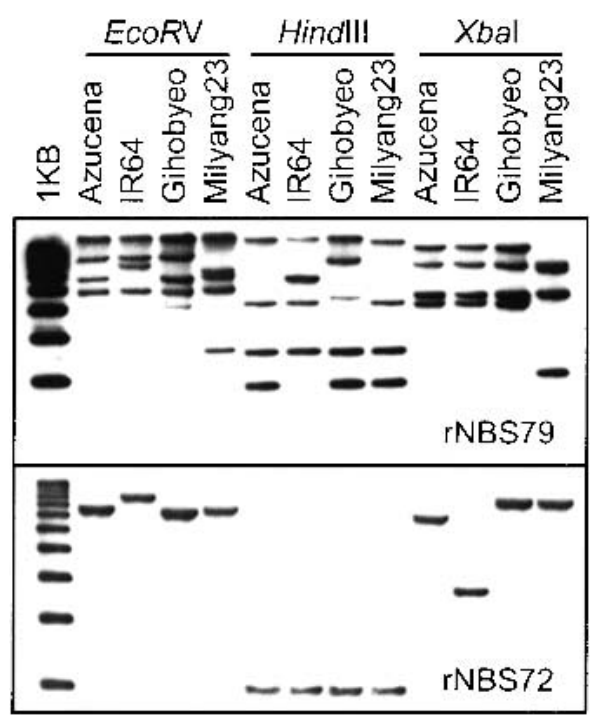

Figure 1 Estimation of genomic copy number of different nucleotide binding site-leucine-rich repeat (NBS-LRR) families in rice. Different cloned NBS-coding sequences were used as probes on gel-blot hybridizations to DNAs of four rice lines digested with four different restriction enzymes. The top blot is probed with rNBS79 and the bottom blot is probed with rNBS72, a predicted single-copy gene. 


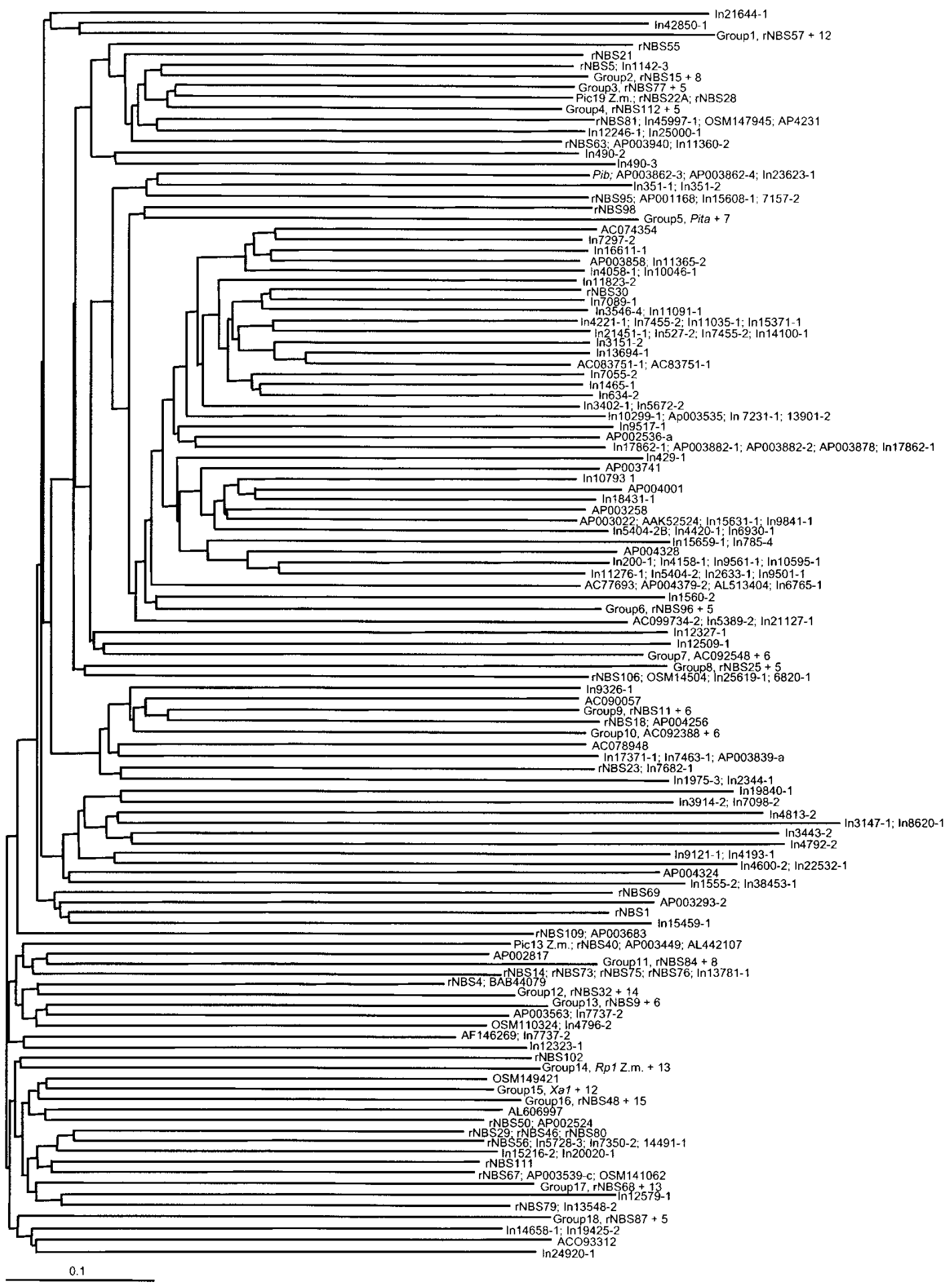

Figure 2 Cladistic analysis of the nucleotide binding site (NBS) region in rice NBS-leucine-rich repeat (LRR) genes. A total of 354 rice sequences and four other cereal R gene sequences were used for generating a neighbor-joining tree. The 354 rice sequences were selected so that none exhibited $>75 \%$ sequence identity to any others in the NBS region. The tree is a representative tree in which groups with $>75 \%$ bootstrapping value (\% of trees of 1000 generated) are represented by a single branch with an average branch length for that group. The complete tree can be viewed from the online supplementary material section. The members of the individual groups are shown in the tree for the smaller groups or listed as follows: Group 1 = rNBS57, In4386-3, In3256-1, In4208-2, In4208-2B, rNBS24, In1277-2, In10933-1, In8374-4, In32618-1, rNBS58, AP003345, In 6313-2; group 2 = rNBS15, rNBS12, rNBS17, rNBS6, rNBS3, rNBS97, In37394-1, In9848-1, AB019186, In6575-2; group 3 = rNBS77, rNBS7, rNBS13, In7573-1, In18748-1, In37595-1; group 4 = rNBS112, rNBS8, OSM13267, In2847-2, In7323-1, AC74283; group 5= Pita, In20736-1, $\ln 1586-1, \ln 10315-1, \ln 20846-1, \ln 3014-1, \ln 785-4 B, \ln 10722-1 ;$ group 6 = rNBS96, AP001073-C, AP001073-a, AP003848, In22736-1, In5389-2; group 7 = AC092548, AP003859, In4930-1, In3292-1, In16749-1, In3436-1, In10436-2; group 8 = rNBS25, rNBS92, AP003918, In31789-1, In40443-1; group 9 = rNBS11, rNBS26, rNBS27, rNBS38, rNBS39, In10362-1; group 10 = AC092388, AC097277-2, AC097277-1, In7217-2, In20247-1, In15637-1, In5597-3; group 11 = rNBS84, AP003568, AP004092, In19349-1, In13218-2, In2456-1, In47000-1, In31232-1, 5790-1; group 12 = rNBS32, rNBS2, rNBS16, rNBS59, rNBS49, rNBS31, rNBS34, rNBS35, rNBS78, AB022164, In7980-1, In10136-1, In2646-2, In10063-1, In340-3; group 13 = rNBS9, rNBS37, In16096-1, In18642-1, In36703-1, In14463-1; group 14 = Rp1, rNBS33, rNBS86, rNBS108, AP004061, AP003368-c, AL606992, AL606616-2, OSM140512, In7903-1, In9457-1, In20413-1, In20680-1; group $15=$ Xa1, rNBS10, rNBS19, rNBS42, rNBS52, rNBS53, AL606660-2, AL606660-5, OSM15716, OSM12815, In12431-1; group 16 = rNBS48, rNBS83, rNBS85, rNBS41, rNBS62, AC098834, AP004010, AP003621-b, OSM15362, OSM14953, OSM11901, In46824-1, In30175-1, In3589-3, In27999-1, In4754-2; group $17=$ rNBS68, rNBS44, AL513004-1, AL513004-2, OSM129685, In271-4, In47803-1, In7388-1, In11951-1, In17986-1, In3169-2, In271-2, In3639-1, In9038-1; group 18 = rNBS87, rNBS36, AP003930-e, AP003930-b, AP003827-d, In1964-1. Gene designations are as in Table 1. 
striction fragments is probably not an accurate reflection of genomic copy number for the genes with several copies. In our experience with the maize $R p 1$ and $R p 3$ gene families, lines with 10 to 20 family members generally show fewer distinct fragments with most enzymes (Webb et al. 2002).

\section{Phylogenetic Analysis of the NBS Region of Rice NBS-LRR Genes}

As mentioned above, the rice sequences were grouped into 354 different families by sequence similarity. The predicted amino acid sequences of the NBS regions of one member of each of these families were aligned for phylogenetic analyses (Fig. 2). Among the rice genes aligned were three conferring known resistance phenotypes: the Xa1 (Yoshimura et al. 1998), Pib (Wang et al. 1999), and Pi-ta (Bryan et al. 2000) genes. Four other cereal genes with demonstrated or suspected resistance phenotypes were also included for comparison; these were barley Mla1 (Zhou et al. 2000), and single members of the maize $R p 1$, putative $R p 3$ (Webb et al. 2002), and PIC19 (Collins et al. 1998) gene families. The different rice sequences formed many distinct clades in the phylogenetic analysis, forming 117 groups when bootstrap values of $>75 \%$ were used to define the groups. Some of the clades were composed of single families or even single genes. For example, the rNBS1 and rNBS69 sequences each form a distinct branch on the tree (Fig. 2) and detect a single gene in gel-blot hybridization experiments (Table 1). Other families formed distinct groups with long branch lengths. These have apparently arisen from ancient duplication events where the different families have diverged considerably in sequence, but still show good homology in conserved regions. Other rice genes are grouped into nondistinct subgroups with different branch lengths indicating a range of different times for the duplication and divergence of their family members. The maize and barley sequences were dispersed on the tree into different clades of rice sequences. Similar results were found when other cereal sequences were included in the analysis (not shown). This would be expected if these groups of resistance genes had already differentiated when the different cereal lineages separated.

\section{Architectural Diversity in Cereal NBS-LRR Genes}

Full-length sequences of cereal NBS-LRR genes were compared to examine their structural diversity. Sequences compared included several cereal genes for which full-length transcripts had been characterized, including the known resistance genes Rp1, Mla1, Xa1, Pib, and Pi-ta, and a full-length rice cDNA AB017914. Full-length transcripts for two additional maize gene families, the putative $r p 3$ family and the PIC19 family, were also isolated. Other sequences included coding regions predicted from genomic sequences. These include annotated sequences obtained from GenBank and gene predictions from genomic sequences by GENSCAN and FGENESH. To represent the full range of diversity of NBS-LRR sequences in rice, we examined a full-length coding sequence for most rice NBS-LRR gene families included in the phylogenetic analysis. Predicted full-length members were identified for $>250$ genes.

\section{$N$-terminal Domain Structures}

Most of the N-terminal regions in the cereal genes ranged from 200-250 amino acids from the start of the coding region to the beginning of the NBS domain (P-loop), similar to most
non-TIR genes in dicots. Because the non-TIR genes from dicots typically have CC motifs, the cereal sequences were examined for this domain structure. Using the COILS program (threshold set to 0.9), CC motifs were apparent in only 47 of the 100 randomly selected sequences. The Paircoil program predicted even fewer CC domains with a threshold of 0.5 . The predicted CC domains were poorly conserved in sequence and in their position, occurring in the beginning, middle, or ends of the N-terminal regions. Most, but not all, of the N-terminal sequences could be aligned reasonably well using alignment programs like ClustalX because of their sequence similarity. To look for conserved aspects of the sequences that were common to all the genes, we examined them with the MEME and Block Maker programs. One conserved sequence motif, designated nT (for non-TIR), was identified, which occurred in nearly all of the rice NBS-LRR genes examined. The nT motif (WVxxIRELAYDIEDIVDxY) was usually located $\sim 130$ amino acids before the P-loop.

The N-terminal region of the $\mathrm{Xa1}$ gene was unusual. Initial analysis indicated the region was relatively long compared to the other genes, coding for a predicted 327 amino acids before the P-loop. A CD (Conserved Domain) search of GenBank (deploys Pfam and Smart databases and NCBI collections) predicted that it codes for a zinc-finger, DNA-binding domain (gnl|Smart|smart00614, score $=69.7$ bits [169], expect $=4 \mathrm{e}-13)$. The zinc-finger domain corresponds to residues $140-188$ of the predicted amino acid sequence, within a relatively typical N-terminal domain and before the NBS domain. Database searches found two other genes with similar amino termini. The genes clustered together with $\mathrm{Xa1}$ in the phylogenetic analyses based on NBS-coding sequences (Fig. 2; sequences rNBS19 and AL606660-5). The two genes flank a gene that is highly similar (presumably allelic) to $\mathrm{Xa1}$ within a 63-kb interval of a BAC clone in the GenBank HTGS database (AL606660). The amino acid sequences of both genes align well with $\mathrm{Xa1}$ but both have diverged, showing only 54\% (rNBS19) and 47\% (AL606660-5) amino acid identity in the NBS region. Both genes were predicted to code for zincfinger, DNA-binding motifs (expected probabilities $=9 \mathrm{e}-07$ and $3 e-06$ ).

\section{The LRR and C-Terminal Regions}

The leucine-rich repeat regions of the rice NBS-LRR genes were quite variable in size and sequence. The repeats in most of the genes were imperfect, with few repeats conforming to a consensus sequence. In some, like Pib (Wang et al. 1999) and $\mathrm{Pi}$-ta (Bryan et al. 2000), the region is leucine-rich but has no clearly distinguishable repeat structure. Roughly one third of the predicted proteins examined were not predicted to have a LRR repeat using the Pfam database. The LRR regions of some of these genes carried $>15 \%$ leucine, but they did not match consensus sequences. When these highly degenerate LRR regions are used to search databases for similar sequences, few, if any, matches are found and similarity is typically weak. At the other extreme, one rice NBS-LRR gene isolated from GenBank (accession no. AP003269) has an LRR region with 16 complete LRRs flanked by two incomplete repeats. Each of the repeats is 24 amino acids long and the positions of the leucine residues in each repeat is highly conserved. The size of the predicted LRR domains was variable, but was between 350 and 700 amino acids for most of the genes.

Several NBS-LRR genes have been identified in Arabidopsis that code for additional domains after the LRR in their C terminus (Dodds et al. 2001; Deslandes et al. 2002). The LRR 
Bai et al.

domains of all the characterized cereal resistance genes extend to the end of the predicted gene products. The coding regions predicted from rice genomic sequences were all similar in this regard. No evidence for any conserved domains following the LRR regions was observed in the rice sequences.

\section{Intron Positions in the NBS Regions}

Introns in the NBS region of cereal NBS-LRR genes have important practical implications for identifying resistance gene sequences by PCR amplification with degenerate primers or identifying them in genomic sequence databases and distinguishing potentially functional genes from pseudogenes. Intron positions can also be used to support phylogenetic interpretation of the relationships between the genes. In a survey of 20 characterized dicot NBS-LRR resistance genes, only the Arabidopsis Rpp8/Hrt gene family had introns in the NBS domain. Three of the characterized cereal resistance genes have introns in their NBS region, that is, Mla1 (Zhou et al. 2000), Pi-ta (Bryan et al. 2000), and Pib (Wang et al. 1999). This suggests that NBS region introns could be more common in cereals. Predicted intron positions in the NBS regions were accordingly examined to estimate their frequency. The NBS regions of several families of genes were verified by amplification and cloning of cDNAs corresponding to genomic sequences that appeared to have introns (Table 2).

The most common intron position in cereals was at the immediate N-terminal side of the kinase-2 motif (Table 2). The Pi-ta, rNBS30, rNBS71, rNBS96, and rNBS98 genes all had introns in this position. These genes all cluster together in the same large group on neighbor-joining trees that were generated based on their NBS sequences, although the bootstrap support for this group is very weak (9\%). This large group is composed of 40 different small clades (rNBS96-AC099734-2; Fig. 2) that contain a total of 87 different gene families. Examination of other representative genes in this group found that the majority of the gene families ( 31 out of 33 examined) have predicted introns in this general position. The 87 gene families in this group represent nearly one fourth of the total rice families (354) that were analyzed on the phylogenetic tree (Fig. 2), consistent with a very ancient origin for this intron position. Further refinement of the relationships between these gene families is needed to determine whether the presence of the intron accurately reflects the evolutionary relationships of these genes.

The rice Pib resistance gene has a single intron between the RNBS-B and GLPL motifs. The position is different than either of the introns in the rNBS102 clade (see below). Two other related genes that form a clade with Pib (Table 2) also have introns in this position. The rNBS84 gene has an intron near the end of the NBS domain, before the conserved MHD motif. This gene occurs in a clade with eight other NBS-LRR genes. Six of the eight genes have sufficient genomic sequence available to determine if introns are present, and all six appear to have introns at this position. Overall, there is relatively good agreement between NBS-area intron positions and the predicted evolutionary relationships among these genes, indicating that intron position can be used to support their classification.

The gene corresponding to rice clone rNBS102 has two introns between the RNBS-B and GLPL motifs. The first intron is located at 13 amino acids after the conserved arginine residue at the end of the RNBS-B motif, and the second one is 11 amino acids before the glycine residue in the GLPL motif (GVPF; Table 2). Genes at the Arabidopsis Rpp8/Hrt locus (McDowell et al. 1998) also have two introns in the NBS region, but the first intron is located before the RNBS-B motif and the second one is 21 amino acids upstream of the glycine residue in the GLPL motif. On this basis, intron positions in the rice rNBS102 gene and the unrelated Arabidopsis Rpp8 gene are not in conserved positions. Surprisingly, no rice genes were identified with intron positions similar to that of the Rpp8 gene. The most similar sequences to $R p p 8$ in the rice databases were AC099402 and OSM144390, neither of which had introns predicted in their NBS domains.

\section{NBS Regions With Unusual Structures}

As described by Wang et al. (1999), the NBS domain of the rice Pib gene has a partially duplicated NBS structure. The gene carries one complete NBS region with an intron between the kinase- 2 and GLPL domains. Directly upstream of this, the

Table 2. Intron Positions in the NBS Domains of Some Cereal NBS-LRR Genes and the Arabidopsis Rpp8 Gene

\begin{tabular}{lll}
\hline Gene & \multicolumn{1}{c}{ General Position } & \multicolumn{1}{c}{ Splicing Position* $^{*}$} \\
\hline rNBS30 & Before kinase-2 & KRYIIVIDDIW \\
rNBS71 & Beginning of kinase-2 & ERYLVIDDIW \\
rNBS96 & Beginning of kinase-2 & KRYLIVIDDLW \\
rNBS98 & Beginning of kinase-2 & RYIVILDDIW \\
Pi-ta & Beginning of kinase-2 & KRYFIIIEDLW \\
rNBS84 & Before MHD & SMVSPVHAKAPRKLTMHDLVYD \\
rNBS102 & Two, between RNBS-B and GLPL & GSKVIVTTRSGAVAKLLGMDLTKPLSSEDCWSLFRRALGVEVKEYNSGDFLDRLKMEVLQKCN \\
RPP8 & Two, 1st between kinase 2 and RNBS-B; & LVVLDDVWKKEDWDVIKAVFPRKRGWKMLLTSRNEGVGIHADPTCLTFRASILNPEESWKLCER \\
& 2nd between RNBS-B and GLPL & IVFPRRETEVRLDEEMEAMGKEMVTHCGGLPLAV \\
Pib-2** & Between RNBS-B and GLPL & TSRIIVTTRKENIANHCSGKNGNVHNLKVLKHNDALCLLSEKVFEEATYLDDQNNPELVKEAKQ \\
Pib-1 & After RNBS-B & ILKKCDGLPL \\
\hline
\end{tabular}

${ }^{*}$ Conserved sequences from NBS domains are underlined. Positions of introns are indicated by boxing the amino acids corresponding to the codon that was created or the two codons that were joined by splicing.

**The Pib gene has a duplicated structure with two tandem, partial NBS regions with introns at essentially the same position in each. Pib-1 indicated the first N-terminal NBS region, which ends before the GLPL domain. 
sequence codes for most of another NBS domain, from the P-loop through the kinase-3a domain until similarity is interrupted by another intron. The structures of the duplications are very similar from the P-loop through the kinase-3a gene where they are flanked on their $3^{\prime}$ by an intron (Table 2), suggesting that the intron may have played a role in creating the duplication. One gene with a similar structure was found in the GenBank HTGS database on a BAC clone (accession no. AP004048). The BAC maps to rice chromosome 2, like Pib, and the predicted protein shows $>90 \%$ sequence identity to that predicted for Pib for much of the coding region. This gene is probably a member of the gene family that the Pib gene detects in gel-blot hybridizations (Wang et al. 1999). Three other genes predicted to code for similar proteins were also identified from a BAC clone (accession no. AP003862) on chromosome 8 . These genes were less related to Pib, only $46 \%-53 \%$ identical in the NBS coding region. Two of the genes had tandem duplications with intron positions like Pib, while the third (AP003862-3) was predicted to code for three tandem duplications separated by introns. Only fragments of this gene were present in the Monsanto and Indica databases, so the structure of this region of the gene could not be verified with a second sequence. The C-terminal-most NBS-coding region, adjacent to the LRR, was typically the most conserved in sequence in all of the genes coding for tandem NBS sequences. The upstream duplicated sequences had typically diverged considerably and were in some cases difficult to recognize by searching for conserved motifs.

\section{A Novel Class of Rice Genes Has No LRR-Coding Region}

While mining NBS-LRR sequences from GenBank, we identified a gene family in rice with a different structure than the known NBS-LRR resistance genes. The most striking difference is that none of the family members possesses an LRR domain. We found a total of 32 genes on five BAC clones from the GenBank database. Eleven of the genes reside on a 202-kb interval spanned by two BAC clones (AC079843 and AC074283) on chromosome 10. The distances between the genes in these two overlapping BACs range from 5.5-52 kb. The first three genes are in the opposite orientation compared to the other eight genes. Another nine clones were closely spaced, in the same orientation, on a 43-kb interval of rice chromosome 1 (AP003292). A single member was found on another BAC clone (AP000570) on chromosome 1. Eleven additional genes were found in the same orientation on a $50-\mathrm{kb}$ interval of a chromosome 7 BAC clone (AP003810). Only one of the genes was interrupted by a gap in the sequence of this clone. Searches of the Monsanto database with these genes identified four additional genes with sequence and structural similarity. A search of the Indica database found 24 genes coding for proteins with $97-100 \%$ sequence identity to those found in the Japonica databases, and these were therefore considered possible alleles. Fourteen additional genes from the Indica database were $<90 \%$ identical to any of the Japonica genes. It is difficult to determine the degree of genomic clustering or the map positions of genes from the Monsanto and Indica databases, as they were identified on smaller, unmapped sequence contigs. In total, 50 genes in this class were identified (Fig. 3). Five partial gene sequences were also observed on some of the smaller sequence contigs that were not identical to any of these 50 , indicating that there are more than 50 genes in this class. The predicted coding regions were composed of single exons and ranged from 385-556 amino acids. One sequence (from Indica contig 32812) was predicted to code for only 258 amino acids, but may be a pseudogene because it appears truncated at both the $\mathrm{N}$ and $\mathrm{C}$ termini. Two other genes from GenBank (AP003292-4 and AC79843-5) and four genes in the Indica database were predicted to have introns, but on closer inspection were found to be likely pseudogenes. At least some of the genes are transcribed, as rice ESTs (BE229855, AU096505, AU166590, AU063352, and AA754293) matching five of the genomic sequences were found in GenBank.

The N-terminus of the non-LRR genes, before the P-loop, consists of roughly 175-210 amino acids in most of the genes. Alignment of the $\mathrm{N}$ termini of these genes found they code for three conserved motifs separated by less conserved regions. To visualize a typical arrangement of conserved motifs, a consensus sequence (Fig. 4) of the gene family was generated from aligned sequences using the program HMMER 2.2g (http:// hmmer.wustl.edu/). The N-terminal sequences show similarity to the $\mathrm{N}$ termini of resistance genes in the non-TIR-NBSLRR genes, but this similarity is very weak; even the conserved $\mathrm{nT}$ motif is poorly conserved. Similar to the regular NBS-LRR genes, the COILs program predicts a CC domain ( $>90 \%$ probability) in some, but not all, of these regions, and the regions coding for predicted CC motifs do not correspond to the conserved motifs.

The NBS coding domain of the rice non-LRR group is also diverged from other NBS-LRR genes. The conserved domain (CD) search tool at NCBI (http://www.ncbi.nlm.nih.gov/) predicts an NBS domain, but sequence similarities are typically weak when compared with other $\mathrm{R}$ gene homologs. When consensus sequences of conserved motifs of NBS-LRR genes are compared to those of the non-LRR gene families, the consensus sequences were slightly different (Table 3 ). Similarity to the NBS-LRR genes falls off shortly after the GLPL motif and the C-terminal $\sim 100$ amino acids of the rice non-LRR genes show little similarity to R genes. One interesting aspect of the NBS domains of the non-LRR rice genes is that some of the motifs that are the most highly conserved among the $\mathrm{R}$ genes are not among the most highly conserved sequences in this gene family. For example, the kinase- 2 and GLPL motifs
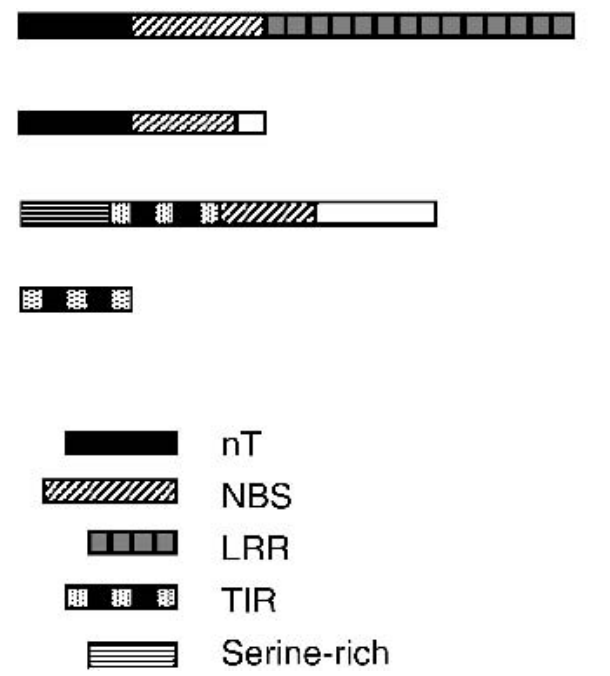

Figure 3 Structures and approximate numbers of nucleotide binding site-leucine-rich repeat and related genes in rice. Open boxes represent domains with no significant database similarity except for similar genes from other plant species. 
Bai et al.

Met11SAvlgDLvSRsISFLIdKYsqqhttsveenleR LqrLLLRvha iVEEAeGRhITNqaMLIQLkmLreaMYR GYYvLDtFryrahqeeaqndeevshsfalskfnpakrl RfstrtrkssaseslsskeLqquLesLEtaisDmkEFV VlLggcPRmsRQPYstYLyl DnCMFGRQvEkEqi InFL LqpdppgaenpgVLPI IGPrrVGKsTLVeHVCnDERVR ShFSsIvflsgDDLedxeemat lrdsgvikHqNpasse RsLvvvElvsDvDeetWrIYSs IrnciarGsKIIIis RsekiarfGTTqalrLkfLskEeYWYFFKVLAFGSadP EEHPkLaSiAmeiAleInGsFiganiladLLRaNfnaq FwsrvLkrlreftkknlslyGeHpkdl lekeshpvyit Rvnsssseylliyddyklceadnasenevpkit fqDll LgsvkppgkFdvLvWrSrIPPYysYvasceilaprhIv rkekrkrsrklree

Figure 4 Consensus sequence of the rice nT-nucleotide binding site (NBS) class genes. The consensus was generated from an alignment of $47 \mathrm{nT}$-NBS genes by HMMER 2.2g. Conserved regions in the $\mathrm{N}$ terminal domain are underlined. Sequences in bold correspond to sequences conserved in NBS-coding domains; the P-Loop, kinase-2, RNBSB, and GLPL motifs (Table 3). More conserved amino acid positions are represented by upper-case letters.

are poorly conserved compared to many other regions of the gene family.

Database similarity (BLASTP) searches of other plant species identified EST sequences from other cereals, such as sorghum (e.g., BM325897) and barley (e.g., AV835233), that code for very similar proteins, but none were apparent among dicot genomic or EST sequences. The most similar Arabidopsis sequences in searches with representative non-LRR members were typically the NBS-LRR genes from the non-TIR class, and these showed weak similarity ( $<25 \%$ identity). For example, a BLASTP search of Arabidopsis proteins using the consensus nT-NBS sequence (Fig. 4) as a query found the best match to be the predicted protein BAB01338, a typical nT-NBS-LRR coding gene. Thus, no evidence of a dicot version of these non-LRR genes was found.

\section{Rice Genes Similar to TIR Coding Sequences}

To determine if the rice genome carries any genes with TIR domains, we searched databases with a consensus TIR sequence designed from the tobacco $N$, flax $L$ and Arabidopsis Rpp5 genes. One sequence was identified in both the Monsanto (OSM12752) and GenBank HTGS (AP003932 and AP003866) databases. A GenScan analysis predicted a coding region composed of three exons coding for 196, 21, and 29 amino acids separated by introns of 121 and 644 nucleotides. A presumably allelic sequence was identified in the Indica database that coded for a protein with only three amino acid differences. An alignment with the TIR domains of $N, L, R P P 5$, TOLL and a human Toll-like receptor gene showed similarity throughout the whole predicted protein (not shown). The sequence apparently represents an expressed gene in cereals, as a barley EST (accession no. BI948029) was identified that was very similar to the rice gene ( $72 \%$ amino acid identity). The gene is also similar (50\% identity for 144 amino acids) to a predicted Arabidopsis protein (accession no. AAG52286). The Arabidopsis protein is also small (199 amino acids) and composed mainly of a TIR domain.

A second class of genes was identified that code for divergent TIR and NBS domains. In total, three genes from this family and one pseudogene were identified. The first was identified in the GenBank (AP000364; protein_id = BAB61209.1) and Monsanto databases (OSM1850). The Indica rice sequence Contig 4057 also codes for most of a protein (except the first 106 amino acids) that is identical in sequence to this predicted protein. A second coding region was carried on overlapping GenBank sequences (AP003256 and AP003274) and on contig OSM15552 and was >99\% identical to a sequence coded by a presumed allele on the Indica sequence Contig2492. An additional gene was coded by the Indica sequence Contig17995, and Contig5477 appears to code for a pseudogene with a stop codon and at least one small deletion. A sequence similar to this latter locus was present in the Japonica rice sequences of the Monsanto database, but it could not be determined if it was a potentially functional allele because the coding region was incomplete. The NBS regions of these genes code for motifs similar to most of the conserved motifs in NBS-coding domains of $\mathrm{R}$ genes (Table 3 ), but their sequences diverge from the $R$ genes after the GLPL motif. The three genes were predicted to encode functional proteins ranging from 986 to 1002 amino acids. The genes code for 165-168 amino acids before their TIR-like domain and this N-terminal region is the least conserved region of the gene (Fig. 5). One feature common to the N-terminal regions of these genes is that they are serine-rich, with $14.5 \%-23 \%$ serine residues. Most of the remainder of the coding regions are more conserved, including the C-terminal 250-300 amino acids domain after the predicted NBS domain. No known protein-coding domains were detected in the C-terminal region, but several highly conserved sequences were apparent among the genes. The C-terminal 275 amino acids of the three genes range from $12 \%-14.5 \%$ leucine. This presents the possibility that these sequences evolved from a degenerate LRR, but the patterns of leucines poorly match that of a leucine-rich repeat.

Database searches identified two Arabidopsis genes that were very similar to the three rice TIR-NBS genes. Sequence homology extends throughout the genes, including the $5^{\prime}$

Table 3. Consensus Sequences of Conserved Motifs in NBS Domains in Different Classes of Genes

\begin{tabular}{llllll}
\hline Gene Class & The P-Loop & \multicolumn{1}{c}{ Kinase-2 } & \multicolumn{1}{c}{ RNBS-B } & GLPL & MHD Domain \\
\hline TIR-NBS-LRR & GIGKTT(il)A & KVL(ivl)(vi)LDDVD & GSRII(iv)TT(qre)D & G(gn)LPLGL & MH(ndkr)LLQQ \\
nT-NBS-LRR & GMGG(vli)GKTTL & (lv)(lvi)(vl)DD(vl)W & GS(kr)(vi)(lva)(fl)T(ts)R & GLRLAL & MHD(vm)(vlim)R(ed) \\
nT-NBS & VGK(skr)TLV & L(vi)(vi)(vi)E(If)xxD & GSK(iv)l(iv)xSR & GSF(ilm)xAN & Not conserved \\
TIR-NBS & SGIGKTEL & L(lv)(iv)IDNL & HV(il)(iv)TTR & GLW(iv)V & Not conserved \\
\hline
\end{tabular}

*Letters in parentheses designate residues that occur in similar frequencies at a single position. Consensus sequences of TIR and Non-TIR genes were calculated from Arabidopsis sequences (http://niblrrs.ucdavis.edu/At_RGenes). The non-LRR consensus sequences were calculated from 47 rice sequences and the TIR-NBS sequences were calculated from three rice genes and two related Arabidopsis genes. 
MegeeesSsfgalpkdpsrnaSSsSI taktsanqspff Spdssnpispaisysps1sSpSkpPssafvsaLqSpyi SpRegePsatvgllPPgetsdpspPlsyssSssfdaPa SsytpPkSdiyt fsdecqsseakasssgsgsgappris Fs fpvpRvSstrqsvsSpvSnnkLRSCDkVy IG fHGqk GaGasLlRFckwLksELElQGIaCFvaDRarysdsqSH ĖadRiicsvtkfGVVVVTrkSFlNPyslEElRFFAGK KNLvPIfFDtgpaeilgRDIVEKRGdLwekhggEkEWV LYGGieKEWkEAvdGLSRVhe fKLEAnEgNWRsCVskT VtvLaskLGRrSiveketkkWRdsegkEelPFPRNrsF VGReKELSElElVLFGngeDvagdserprgelktkerr SGkskdGladeeSdtVrTrngKfisleLwKcsepei Ea QsdpIekspgrgrnl lkqrskgrksRkkrrssKslygk GVACi SGESGIGKTELALEFAYRYSQRYKMVLWIGGEa RYIRQNYLNLSKMYL.glDi.SaeaekerGRIRSFEEQEE EAfarIkrELfRDi PyLliIDNLEsEKDWWegKkdlhD LiPRntGgTHviITTRLPrVMN1EPmgLsyLSgaeAMi LmkGkvKkDYPveEievLkrki dEKLGRLS IGLwiVGS LLSELpItPSrLleavnriplremslsLganDenll kn KNeFLikvLvfCfai fDhAdGprsLAtrMvvaggwLAP APVPstLLAlaAhKlPtKhrgkpkllwrsLkraltCGt HCflasrskRSEaEAAlLLvRlglaRkt srkPGCWIQF Hpi tqLkfARkRgglapatAaVqgVirrgnisvysdHL WA saFLVFGFkSEPPIVQLkasdlvl fikKqtaLPLAI rtFiTFSRCnSALELLkVCTNVLEeaekS1vSriqdwr qgsLlCwkkklotnkaqvdEylWQEvtLLKAt ILETRAK LLLRGGqFDtGeeLiRtc Is IRTVml GhdHpnTlaAkq ETLaKLvRliskiqliHntsPk

Figure 5 Consensus sequence of three predicted rice TIRnucleotide binding site (NBS) genes with two related Arabidopsis proteins. The region showing similarity to the TIR domain of TIR-NBSleucine-rich repeat genes is underlined. Sequences in bold correspond to sequences conserved in NBS-coding domains, the P-Loop, kinase-2, RNBSB, and GLPL motifs (Table 3). Serine residues in the serine-rich $\mathrm{N}$ terminus and leucine residues in the $\mathrm{C}$ terminus are highlighted. More conserved amino acid positions are represented by upper-case letters.

and 3 ' regions. The sequence affinities of the five genes indicate that the ancestral gene had duplicated and the two paralogs had diverged before monocots and dicots split. The predicted proteins of the two rice genes from AP000364 and Contig17995 are more similar to the Arabidopsis At5g56220 protein (59\% and 55\% identical, respectively) than they are to the rice AP003256 protein (both 36\% identical). Similarly, the other rice protein (from AP003256) is more similar (53\% identical) to the other Arabidopsis protein, At4g23440.

\section{A Phylogeny of Rice Genes Based on NBS Region Sequences}

To examine the evolutionary relationships of the different types of NBS-coding sequences in rice, the sequences of the rice nT-NBS genes and TIR-NBS genes were compared to representative NBS-LRR genes from rice and other species (Fig. 6). The rice NBS-LRR genes were selected to represent a diversity of different clades from the previous analysis of these genes (Fig. 2). Amino acid sequences utilized from the nT-NBS and TIR-NBS genes were limited to the region between the P-loop to the GLPL motif because of the limited identity between classes outside this region. For comparative purposes, several NBS-LRR resistance genes from dicots also were used, including five representative genes from the TIR class (Arabidopsis Rpp1, flax $L 6$ and $M$, and tobacco $N$ ) and four genes from the non-TIR subclass (Arabidopsis Rps5 and Rpm1, tomato $I 2$ and potato $R x$ ). Two Arabidopsis genes that were related to the rice TIR-NBS genes also were included. Trees based on distance and parsimony had similar topologies.

The rice and Arabidopsis TIR-NBS genes formed a distinct clade that was well separated from NBS-LRR genes. It was separated into two distinct groups, each with an Arabidopsis gene and one or two rice genes. This supports the hypothesis that there were at least two genes of this class in the nearest common ancestor of modern monocots and dicots. The rice nT-NBS genes also formed a single large clade that was distinct from the NBS-LRR genes. The variation within this group and the deep divisions between the different subgroups indicated that the nT-NBS genes comprise a very old gene family, like the NBS-LRR genes. The different subgroups are, at least partially, separated into different chromosomal regions in the rice genome. One group is composed mainly of sequences from the two overlapping chromosome 10 BACs (AC79843 and AC074283), while the clones from a chromosome one BAC (AP003292) all belonged to a separate group. Similarly, the sequences from the chromosome 7 BAC clone (AP003810) also cluster together in $69 \%$ of the trees (Fig. 6).

The cereal and dicot NBS-LRR genes formed two distinct groups, as expected from previous analyses that found the TIR subclass to be a distinct group (Meyers et al. 1999; Pan et al. 2000b; Young 2000). The four non-TIR class dicot R genes included in the analysis did not cluster together, but rather grouped with different cereal genes with bootstrap values ranging from $71 \%$ (for potato $R x$ ) to $100 \%$ (for Arabidopsis Rps5). This agrees with the proposal by Cannon et al. (2002) that there are several ancient sequence clades of nT-NBS-LRR genes and that some of these predate the split between monocots and dicots.

\section{Similarity Between Rice NBS-LRR Genes and Those From Other Cereals}

To estimate the extent to which the genomic sequences include most NBS-LRR gene families, we selected 61 rice gene fragments that potentially code for partial NBS-coding domains from GenBank and used to search the available genomic sequences. Forty-nine (80\%) of the NBS gene fragments matched highly similar genomic sequences $(\geq 95 \%$ amino acid identity) and all but one matched sequences with $>85 \%$ sequence identity. The remaining sequence identified a less closely related family member, with $77 \%$ amino acid identity. This indicates that members of nearly all of the NBSLRR families are represented in the available genomic sequences, although the gene fragments used in the similarity searches are clearly not a random sample of the NBS-LRR genes. The rice sequences in our clone collection and in the GenBank, Monsanto, and Indica databases should therefore represent most of the NBS-LRR genes in rice, or at least carry members of most NBS-LRR gene families.

A similar approach was used to examine the extent of similarity of rice NBS-LRR genes to those of other cereal species. Forty-seven fragments that potentially code NBS genes from different cereal species were used to identify the most similar rice sequences in the genomic sequence databases (Table 4). Comparisons of the 47 sequences to one another revealed 28 groups or families with $>75 \%$ amino acid identity within the families. The different members of these groups 
Bai et al.

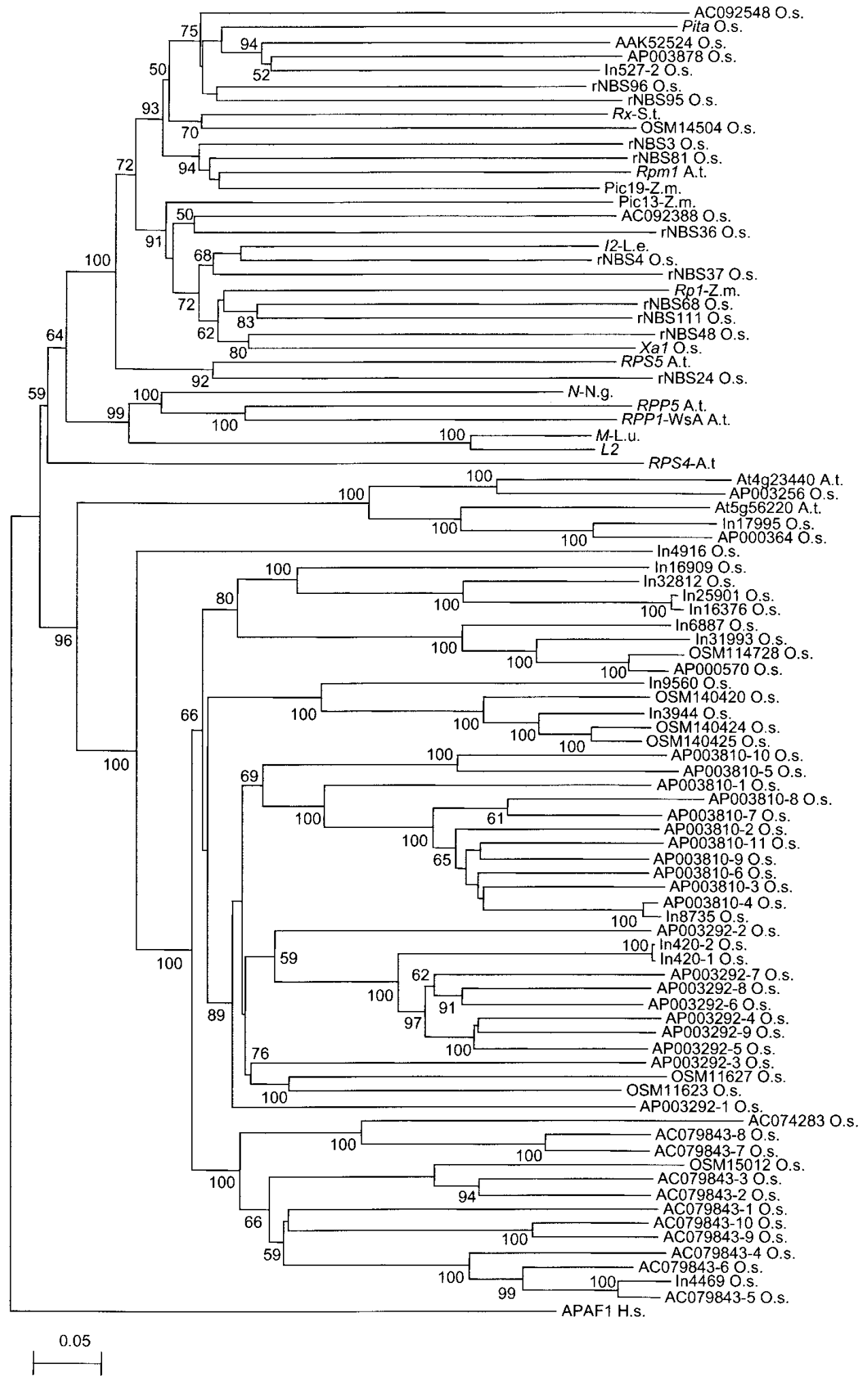

Figure 6 Phylogenetic analysis of nucleotide binding site-leucine-rich repeat (NBS-LRR) and related genes from rice and dicot species. Rice NBS-LRR sequences were selected from different clades identified in Figure 1. Other designations correspond to characterized resistance genes or GenBank, Monsanto, or Indica database accession numbers and the species designations are indicated after the clone designation: O.s., rice; Z.m. maize; S.t. potato; A.t. Arabidopsis; L.e. tomato; L.u. flax; N.g. tobacco, and H.s. human. The $48 \mathrm{nT}-\mathrm{NBS}$ sequences are all from rice, while two of the TIR-NBS sequences are from Arabidopsis and the other three are from rice.

\section{Genome Research}


generally showed similar levels of identity to the same rice sequence. The different cereal genes exhibited a range of sequence similarities to the rice genes. Two of the maize sequences (mNBS2 and AF056161) showed 84\% and 85\% amino acid identity with rice sequences, and one wheat (AF087521) sequence showed 85\% identity. Alternatively, many of the 28 families did not identify likely orthologs. Surprisingly, 10 of the 28 families showed amino acid identities of $\leq 60 \%$.

\section{DISCUSSION}

As the largest class of disease resistance genes, the NBS-LRR genes play a critical role in defending plants from a multitude of pathogens and pests. The availability of nearly complete genomic sequences of two distantly related plant species, rice and Arabidopsis, allows comparative evolutionary analyses of these genes. Previous analyses of available cereal sequences have implied that the cereal NBS-LRR genes may be more homogeneous in their domain architecture than similar genes in dicots. The dicot genes can be divided into two distinct groups, those coding for a TIR domain at their N-termini and those without the TIR domain. The TIR class NBS-LRR genes account for the largest proportion of the NBS-LRR genes in the Arabidopsis genome (http://niblrrs.ucdavis.edu/ At_RGenes/), but this class has not yet been found in cereals. We failed to detect these sequences after examining roughly $820 \mathrm{Mb}$ of assembled rice genomic sequence from two different genotypes of the estimated $430-\mathrm{Mb}$ rice genome. They also are absent in cereal EST databases. It therefore seems the TIR class is not only rare in cereals, but probably absent. The presence of these sequences in gymnosperm databases, like the Pinus taeda EST sequence database (e.g., GenBank accession no. BI077056) indicates that this class of gene was present in the progenitors of grass species, but lost in the grass family (Meyers et al. 2002). It is likely that there were a very small number of TIR class genes in early angiosperms, and that their numbers amplified in the progenitors of modern dicots as they became more dependent on them for defense against pathogens. On the other hand, our results and those of others (Cannon et al. 2002) have shown that specific monocot and dicot nT-NBS-LRR genes cluster together in phylogenetic analysis as expected if several members of this class had already diverged in early angiosperms. The numbers of genes in this class has amplified to $\sim 600$ or more in rice, compared to $\sim 50$ in Arabidopsis (http://niblrrs.ucdavis.edu/ At_RGenes/).

One class of NBS-LRR-related gene was identified in cereals that may be specific to cereal, or monocot genomes. The nT-NBS class shows similarity to the N-terminal half of nTNBS-LRR genes but has no LRR domain. Other genes related to nT-NBS-LRR genes without LRR domains were observed in cereal genomes and are also present in Arabidopsis (Meyers et al. 2002). For example, the $r p 1-p d 5$ gene is a transcribed member of the $R p 1$ family of maize and is $99 \%$ identical to the Rp1-D gene, but is truncated before the LRR coding region (Sun et al. 2001). The nT-NBS family is different from these sequences in several respects. As a group, their sequences have diverged considerably from the other NBS-LRR genes, evidence that they have evolved independently from these genes for most of their evolution. The family is also different in that it is monophyletic and contains no known members that code for LRR domains. Although the family appears very rare, or missing, in dicot genomes, it is a very old gene family as
Table 4. Sequence Similarity Between Rice Sequences and Genes From Other Cereal Species

\begin{tabular}{|c|c|}
\hline Cereal NBS Family* & Most Similar Rice Sequence ${ }^{* *}$ \\
\hline mNBS1 Zea & AF032688 84\% \\
\hline mNBS2 Zea & rNBS6, In37394 71\% \\
\hline mNBS6 Zea & $\ln 757364 \%$ \\
\hline AF056150 Zea & $\ln 757359 \%$ \\
\hline AF056152 Zea & $\ln 5523475 \%$ \\
\hline AF056155 Zea & AP003914, In20630 58\% \\
\hline AF056161 Zea & AP003280, $\ln 93085 \%$ \\
\hline AF107293 Zea (Rp1) & AP003368 72\% \\
\hline AF056153 & AP003449, In17424 74\% \\
\hline AF056159 & $\ln 161356 \%$ \\
\hline AF325196 Triticum & $\ln 161359 \%$ \\
\hline \multirow{6}{*}{\multicolumn{2}{|c|}{$\begin{array}{l}\text { AF320290 (85\%) Secale } \\
\text { AF108008 (81\%) Hordeum } \\
\text { AF108011 (81\%) Hordeum } \\
\text { AF108012 (83\%) Hordeum } \\
\text { AF108013 (81\%) Hordeum } \\
\text { AF108015 (80\%) Hordeum }\end{array}$}} \\
\hline & \\
\hline & \\
\hline & \\
\hline & \\
\hline & \\
\hline AF108010 Hordeum & $\ln 732365 \%$ \\
\hline AF078873 Avena & $\ln 4386$ 78\% \\
\hline AF032679 (84\%) Hordeum & \\
\hline AF032682 Hordeum & AF032688 81\% \\
\hline \multicolumn{2}{|l|}{ AF087519 (91\%) Triticum } \\
\hline \multicolumn{2}{|l|}{ AJ296001 (87\%) Avena } \\
\hline AF032684 Hordeum & In8824 (62\%) \\
\hline AF032686 Hordeum & $\ln 1031560 \%$ \\
\hline AF032687 Hordeum & AP003948, AP003914 67\% \\
\hline AJ302298 Hordeum & $\ln 2380855 \%$ \\
\hline \multicolumn{2}{|l|}{ AY009939 (84\%) Hordeum } \\
\hline \multicolumn{2}{|l|}{ A)302296 (77\%) Hordeum } \\
\hline AF032685 Hordeum & AC099734 61\% \\
\hline AF149114 Triticum & $\ln 542960 \%$ \\
\hline \multirow{2}{*}{\multicolumn{2}{|c|}{$\begin{array}{l}\text { AF052641 Triticum } \\
\text { AF158635 (82\%) Triticum }\end{array}$}} \\
\hline & \\
\hline AF158634 Triticum & In7095 59\% \\
\hline AF087521 Triticum & $\ln 2088985 \%$ \\
\hline AF325198 Triticum & $\ln 161359 \%$ \\
\hline \multirow{2}{*}{\multicolumn{2}{|c|}{$\begin{array}{l}\text { A)295999 Avena } \\
\text { TVE249945 } 73 \% \text { Aegilops }\end{array}$}} \\
\hline & \\
\hline \multicolumn{2}{|l|}{ TVE249944 74\% Aegilops } \\
\hline \multicolumn{2}{|l|}{ AF087518 74\% Triticum } \\
\hline \multicolumn{2}{|l|}{ AF087520 81\% Triticum } \\
\hline \multirow{2}{*}{\multicolumn{2}{|c|}{ AF032683 78\% Hordeum }} \\
\hline & \\
\hline A)249948 Aegilops & In6820 63\% \\
\hline AJ249949 Aegilops & $\ln 682066 \%$ \\
\hline $\begin{array}{l}\text { A|249943 Aegilops } \\
\text { A|296002 } 79 \% \text { Avena }\end{array}$ & $\ln 1036270 \%$ \\
\hline A|295998 Avena & AF032692 64\% \\
\hline
\end{tabular}

${ }^{*}$ Cereal sequence, or family of related sequences, with a predicted NBS coding region. When families of related sequences are listed, the top sequence shows the highest identity to a rice sequence. Numbers in parentheses next to other sequences give their identity (\% amino acid) to the top family member.

${ }^{* *}$ Accession no. of the rice sequence that exhibits the highest sequence identity. Accession nos. starting with ' $A$ ' are GenBank accessions, and an 'In' prefix indicates the Indica sequence database. Percentages indicate the $\%$ amino acid identity for at least 155 amino acids, typically from the P-loop to the GLPL domain. Cereal sequences that did not include this entire region were not examined.

evidenced by the extensive sequence divergence among members. Over 50 members exist in rice, making the family roughly the size of the nT-NBS-LRR family in Arabidopsis.

Some limited structural heterogeneity was observed in 
the cereal NBS-LRR genes as several genes with duplicated or novel domains were observed. For example, the $\mathrm{Xa1}$ gene carries sequences near its $\mathrm{N}$ terminus that are predicted to code for a zinc-finger, DNA-binding domain. The $\mathrm{N}$ termini of the vast majority of the N-terminal domains were quite homogeneous, that is, they were typically small with at least one highly conserved region. Many code for predicted CC domains, although this is not a distinguishing feature of this class of genes. While the structures of these genes are fairly conserved, they are extremely diverse in sequence. The Cterminal regions of many of the genes are barely recognizable as LRR domains. Even the NBS regions of the genes have diverged extensively and classification of the genes based on the sequence of this region reveals over 100 distinct clades. Some of these clades consist of one or a few genes in the rice genome, while others have amplified into large groups with varying degrees of similarity. Much of the divergence among these genes apparently occurred before the different cereal species separated, as NBS coding sequences of other cereals typically cluster within the rice clades.

While typical TIR class NBS-LRR genes do not appear to be present in cereal species, genes related to this class are present. One rice gene was identified, with strong homology to a barley EST, which coded for a TIR domain but no other recognizable domains. A second class, with at least three genes in rice, coded for divergent TIR and NBS domains. The C-terminal domain of these genes may have been derived from an LRR-like domain but was unique. Homologs of both of these classes of genes were observed in the Arabidopsis genome. In fact, sequence affinities between the rice TIR-NBS genes, and two similar Arabidopsis genes provide evidence that two members of this gene class were present when monocots and dicots diverged.

The structural differences between NBS-LRR genes in Arabidopsis are partially correlated with their dependence on certain other disease-signaling components (Glazebrook 2001; Austin et al. 2002). For example, a functional Eds1 gene is required for resistance mediated by the TIR-NBS-LRR genes Rps4 and Rpp5 but is not required for several non-TIR class genes, such as Rps 2 and Rpm1 (Aarts et al. 1998). The predominance of the non-TIR class in cereals might indicate that the cereal R genes signal through fewer or simpler pathways. On the other hand, the Mla1 and Mla6 genes are highly similar in sequence and structure, but differ in their requirements for the Rar1 and Sgt1 gene products (Zhou et al. 2000; Azevedo et al. 2002; Halterman et al. 2002). It seems likely, therefore, that the different defense signaling pathways that cereal $\mathrm{R}$ genes utilize depend on factors other than obvious differences in structural domains.

NBS-coding sequences from other cereals exhibit a surprising range of similarity to the rice sequences. Some maize and wheat sequences exhibit $85 \%$ amino acid sequence identity to rice genes, while 10 of 28 families showed $<60 \%$ sequence identity. It is possible that the rice orthologs of some of these families are missing from the available rice databases, but most of the rice genes are recently duplicated, and it is unlikely that all the sequences would be missing for very many families. This would also explain why we and others (Leister et al. 1998), have found that many of the rice sequences cross-hybridize weakly to other cereal species. This may be an indication that the resistance genes are evolving at very different rates. Alternatively, it could be from loss of resistance genes, or gene families in different species lineages (Michelmore and Meyers 1998; Cannon et al. 2002). If resistance genes are commonly lost from species lineages, comparative mapping experiments might frequently mistake similar sequences for orthologs when they are actually more distantly related paralogs. This may explain why the initial comparative mapping experiments with resistance genes in cereals have implied that their relative map positions may be less conserved than other types of genes (Leister et al. 1998). The present collection of NBS coding clones should provide sufficient probes for more detailed comparative mapping experiments, allowing a more extensive test of relative levels of

Table 5. List of Bioinformatics Programs Used in This Study

\begin{tabular}{|c|c|c|c|}
\hline Program & Functions Used In This Study & Web Site URL & References \\
\hline Clustal X & $\begin{array}{l}\text { Multiple sequence alignment; } \\
\text { Bootstrapping analysis }\end{array}$ & ftp://ftp-igbmc.u-strasbg.fr/pub/ClustalX/ & $\begin{array}{l}\text { Thompson et al. } 1997 \text {, } \\
\text { Jeanmougin et al. } 1998\end{array}$ \\
\hline GeneDoc & Editing aligned sequences & http://www.psc.edu/biomed/genedoc/ & Nicholas et al. 1997 \\
\hline GENSCAN & Predicting open-reading-frames & http://genes.mit.edu/GENSCAN.html & Burge and Karlin 1997 \\
\hline FGENESH & Predicting open-reading-frames & http://genomic.sanger.ac.uk/ & Solovyev V.V. 2001 \\
\hline PAUP & Parsimony tree generation & http://paup.csit.fsu.edu & Swofford, D.L. 2002 \\
\hline Pfam & $\begin{array}{l}\text { Conserved protein domain } \\
\text { search }\end{array}$ & http://pfam.wustl.edu/ & Bateman et al. 2002 \\
\hline Smart & $\begin{array}{l}\text { Conserved protein domain } \\
\text { search }\end{array}$ & http://smart.embl-heidelberg.de/ & Letunic et al. 2002 \\
\hline MEME & $\begin{array}{l}\text { Conserved protein domain } \\
\text { search }\end{array}$ & http://meme.sdsc.edu/meme/website/meme.html & Bailey et al. 1999 \\
\hline MAST & $\begin{array}{l}\text { Conserved protein domain } \\
\text { search }\end{array}$ & http://meme.sdsc.edu/meme/website/mast-intro.html & Bailey et al. 1999 \\
\hline Block Maker & $\begin{array}{l}\text { Conserved protein domain } \\
\text { search }\end{array}$ & http://www.blocks.fhcrc.org/blockmkr/ & Henikoff et al. 1995 \\
\hline TreeView & $\begin{array}{l}\text { Phylogenitic tree image } \\
\text { generation }\end{array}$ & http://taxonomy.zoology.gla.ac.uk/rod/treeview.html & Page 1996 \\
\hline NJPlot & $\begin{array}{l}\text { Phylogenitic tree image } \\
\text { generation }\end{array}$ & http://pbil.univ-lyonl.fr/software/njplot.html & Perrière and Gouy 1996 \\
\hline COILS & Predicting coiled-coil domain & http://www.ch.embnet.org/software/COILS_form.html & Lupas et al. 1991 \\
\hline Paircoil & Predicting coiled-coil domain & http://nightingale.Ics.mit.edu/cgi-bin/score & Berger et al. 1995 \\
\hline
\end{tabular}

1882 Genome Research

www.genome.org 
synteny. Examination of the presence or absence, and estimation of copy numbers of the different NBS-LRR gene families in the different grass species will shed light on the evolutionary dynamics of resistance-gene gain and loss in cereal genomes. The sequences identified in the present study provide a framework for classification of additional cereal genes.

\section{METHODS}

\section{Sequence Acquisition}

Cereal resistance gene sequences were obtained either from cloned PCR products (below) or by searching various databases with amino acid sequences of specific resistance genes as queries. Initial queries were performed with known resistance genes. Additional queries were done with more unique sequences after initial cladistic analyses. Two things that were considered do determine whether sequences were retained for phylogentic and structural analysis: (1) whether they were NBS-LRR sequences as indicated by conserved domains (domains in Table 3); legitimate NBS-LRR genes often had $<30 \%$ amino acid identity to the sequences used in the initial queries and sometimes had TBLASTN scores $<1 \mathrm{e}-4$, but sequences with lower scores were often apparent pseudogenes with interrupted coding regions; and (2) whether they were $<75 \%$ identical in amino acid sequence to already collected sequences. To determine this, a local database of the predicted proteins of these sequences was sequentially searched by BLASTP with each of the new sequences. This allows the identification of identical sequences and their classification into families. The local database was updated with the new sequences periodically. Databases searched include the GenBank nonredundant and high-throughput genomic sequence databases (http://www.ncbi.nlm.nih.gov/blast/index.html), the Monsanto rice genomic database (www.rice-research.org), and the Indica rice genomic database posted by the Beijing Genomics Institute (http://btn.genomics.org.cn/rice). The final search to above-mentioned databases was in May 2002.

\section{Cloning and Characterization of Maize and Rice NBS Region Sequences}

Primers were designed from the NBS-coding sequences obtained from the database searches. Forward primers were produced in such a way that they ended at the beginning of the P-loop of the NBS region of each gene. The reverse primers were designed about $900 \mathrm{bp}$ after the P-loop, and at the end of the NBS region where the amino acid sequence 'MHD' is moderately conserved. The PCR products that showed $0.9-1 \mathrm{~kb}$ in size were cloned into pCR2.1-TOPO cloning vector from Invitrogen and sequenced in the Kansas State University DNA sequencing facilities. Maize inbred line B73 and an Indica rice variety Nipponbare were used to generate RNA or DNA templates. Genomic DNA was usually used as a template, but RNA was used to amplify several NBS sequences to verify intron positions.

Full-length coding sequences were isolated from two previously isolated maize NBS clones, PIC13 and PIC19. Genomic $\lambda$ clones homologous to the probes were isolated from libraries made from the maize lines B73 (for PIC19) and Rp3$A$-R168 (PIC13). The PIC13 probe is thought to represent a member of the Rp3 gene family (Collins et al. 1998; Webb et al. 2002). The NBS-LRR genes were sequenced, following subcloning, into a pUC19 vector. Transcripts corresponding to the genes were isolated by RACE PCR using $5^{\prime}$ and $3^{\prime}$ RACE System For Rapid Amplification of cDNA Ends from Gibco Invitrogen Corporation.

\section{Genomic Hybridizations}

Cloned fragments of rice NBS-LRR genes were used as probes on genomic blots of rice to estimate the genomic copy num- bers of each of the different families. Five micrograms of genomic DNA from four rice cultivars (Azucena, IR64, Gihobyeo, and Milyang23) were digested with four restriction endonucleases (EcoRI, EcoRV, HindIII, and $\mathrm{XbaI}$ ), separated on $0.8 \%$ TBE agarose gels and blotted prior to hybridization. Probe labeling, hybridization, and signal detection were performed using ECL Direct Nucleic Acid Labeling And Detection System from Amersham Pharmacia Biotech. Blots were washed following hybridization at a moderate hybridization stringency of $0.5 \mathrm{X}$ SSC $(75 \mathrm{mM} \mathrm{NaCl}$ and $7.5 \mathrm{mM}$ sodium citrate) at $65^{\circ} \mathrm{C}$.

\section{Bioinformatic Programs Used for Phylogenetic Studies}

The bioinformatic programs used in this study are listed in Table 5. All parameters in these programs were set to default except that 'Arabidopsis' was specified as the organism in GenScan, and 'Monocots' was specified in FGENESH.

\section{ACKNOWLEDGMENTS}

The authors wish to thank Blake Meyers and Richard Michelmore for valuable discussions. This work was supported by NSF Plant Genome grant 9975971.

The publication costs of this article were defrayed in part by payment of page charges. This article must therefore be hereby marked "advertisement" in accordance with 18 USC section 1734 solely to indicate this fact.

\section{REFERENCES}

Aarts, N., Metz, M., Holub, E., Staskawicz, B.J., Daniels, M.J., and Parker, J.E. 1998. Different requirements for EDS1 and NDR1 by disease resistance genes define at least two $R$ gene-mediated signaling pathways in Arabidopsis. Proc. Natl. Acad. Sci. 95: 10306-10311.

Austin, M.J., Muskett, P., Kahn, K., Feys, B.J., Jones, J. D., and Parker, J.E. 2002. Regulatory role of SGT1 in early R gene-mediated plant defenses. Science 295: 2077-2080.

Azevedo, C., Sadanandom, A., Kitagawa, K., Freialdenhoven, A., Shirasu, K., and Schulze-Lefert, P. 2002. The RAR1 interactor SGT1, an essential component of R gene-triggered disease resistance. Science 295: 2073-2076.

Bailey, T.L., Baker, M.E., Elkan, C.P., and Grundy, W.N. 1999. MEME, MAST, and Meta-MEME: New tools for motif discovery in protein sequences. In Pattern discovery in biomolecular data: Tools, techniques, and applications. (eds. J. Wang, B. Shapiro, and D. Shashas), pp. 30-54. Oxford University Press, Oxford, UK.

Bateman, A., Birney, E., Cerruti, L., Durbin, R., Etwiller, L., Eddy, S.R., Griffiths-Jones, S., Howe, K.L., Marshall, M., and Sonnhammer, E.L.L. 2002. The Pfam protein families database. Nucleic Acids Res. 30: 276-280.

Bent, A.F. 1996. Plant disease resistance genes: Function meets structure. Plant Cell 8: 1757-1771.

Berger, B., Wilson, D.B., Wolf, E.T.T., Milla, M., and Kim, P.S. 1995. Predicting coiled coils by use of pairwise residue correlations. Proc. Natl. Acad. Sci. 92: 8259-8263.

Bryan, G.T., Wu, K.-S., Farrall, L., Jia, Y., Hershey, H.P., McAdams, S.A., Donaldson, G.K., Tarchini, R., and Valent, B. 2000. A single amino acid difference distinguishes resistant and susceptible alleles of the rice blast resistance gene Pi-ta. Plant Cell 12: 2033-2046.

Burge, C. and Karlin, S. 1997. Prediction of complete gene structures in human genomic DNA. J. Mol. Biol. 268: 78-94.

Cannon, S.B., Zhu, H., Baumgarten, A.M., Spangler, R., May, G., Cook, D.R., and Young, N.D. 2002. Diversity, distribution, and ancient taxonomic relationships within the TIR and Non-TIR NBS-LRR resistance gene subfamilies. J. Mol. Evol. 54: 548-562.

Collins, N.C., Webb, C.A., Seah, S., Ellis, J.G., Hulbert, S.H., and Pryor, A. 1998. The isolation and mapping of disease resistance gene analogs in maize. Mol. Plant Microbe Interact 11: 968-978.

Deslandes, L., Olivier, J., Theulieres, F., Hirsch, J., Feng, D.X., 
Bai et al.

Bittner-Eddy, P.D., Beynon, J., and Marco, Y. 2002. Resistance to Ralstonia solanacearum in Arabidopsis thaliana is conferred by the recessive RRS1-R gene, a member of a novel family of resistance genes. Proc. Natl. Acad. Sci. 99: 2404-2409.

Dodds, P.N., Lawrence, G.J., and Ellis, J.G. 2001. Six amino acid changes confined to the LRR B-strand/B-turn motif determine the difference between the $\mathrm{P}$ and Ps rust resistance specificities in flax. Plant Cell 13: 163-178.

Ellis, J., Dodds, P., and Pryor, T. 2000. The generation of plant disease resistance gene specificities. Trends Plant Sci. 5: 373-379.

Ellis, J.G., Lawrence, G.J., Luck, J.E., and Dodds, P.N. 1999. Identification of regions in alleles of the flax rust resistance gene $\mathrm{L}$ that determine differences in gene-for-gene specificity. Plant Cell 11: 495-506.

Glazebrook, J. 2001. Genes controlling expression of defense responses in Arabidopsis-2001 status. Current Opinion in Plant Biology 4: 301-308.

Goff, S.A., Ricke, D., Lan, T.H., Presting, G., Wang, R., Dunn, M., Glazebrook, J., Sessions, A., Oeller, P., Varma, H., et al. 2002. A draft sequence of the rice genome (Oryza sativa L. ssp. japonica) Science 296: 92-100.

Halterman, D.A., Zhou, F., Wei, F., Wise, R., and Schulze-Lefert, P. 2002. The MLA6 coiled-coil, NBS-LRR protein confers AvrMla6-dependent resistance specificity to Blumeria graminis $\mathrm{F}$. sp. hordei in barley and wheat. Plant J. 25: 335-348.

Hammond-Kosack, K.E. and Jones, J.D.G. 1997. Plant disease resistance genes. Annual Review of Plant Physiology and Plant Molecular Biology 48: 575-607.

Henikoff, S., Henikoff, J.G, Alford, W.J, and Pietrokovski, S. 1995. Automated construction and graphical presentation of protein blocks from unaligned sequences. Gene 163: GC17-GC26.

Hulbert, S.H., Webb, C.A., Smith, S.M., and Sun Q. 2001. Resistance gene complexes: Evolution and utilization. Annu. Rev. Phytopathol. 39: 285-312.

Jeanmougin, F., Thompson, J.D., Gouy, M., Higgins, D.G., and Gibson, T.J. 1998. Multiple sequence alignment with Clustal X. Trends Biochem. Sci. 23: 403-405.

Leister, D., Kurth, J., Laurie, D.A., Yano, M., Sasaki, T., Devos, K. Graner, A., and Schulze-Lefert, P. 1998. Rapid reorganization of resistance gene homologues in cereal genomes. Proc. Natl. Acad. Sci. 95: 370-375.

Letunic, I., Goodstadt, L., Dickens, N.J., Doerks, T., Schultz, J., Mott, R., Ciccarelli, F., Copley, R.R., Ponting, C.P., and Bork, P. 2002. Recent improvements to the SMART domain-based sequence annotation resource. Nucleic Acids Res. 30: 242-244.

Luck, J.E., Lawrence, G.L., Dodds, P.N., Shepherd, K.W., and Ellis, J.G. 2000. Regions outside of the leucine-rich repeats of flax rust resistance proteins play a role in specificity determination. Plant Cell 12: 1367-1377.

Lupas, A., Van Dyke, M., and Stock, J. 1991. Predicting coiled coils from protein sequences. Science 252: 1162-1164.

McDowell, J.M., Dhandaydham, M., Long, T.A., Aarts, M.G., Goff, S., Holub, E.B., and Dangl, J.L. 1998. Intragenic recombination and diversifying selection contribute to the evolution of downy mildew resistance at the RPP8 locus of Arabidopsis. Plant Cell 10: $1861-1874$

Meyers, B.C., Shen, K.A., Rohani, P., Gaut, B.S., and Michelmore, R.W. 1998. Receptor-like genes in the major resistance locus of lettuce are subject to divergent selection. Plant Cell 11: $1833-1846$.

Meyers, B.C., Dickerman, A.W., Michelmore, R.W., Pecherer, R.M., Sivaramakrishnan, S., Sobral, B.W., and Young, N.D. 1999. Plant disease resistance genes encode members of an ancient and diverse protein family within the nucleotide-binding superfamily. Plant J. 20: 317-332.

Meyers, B.C., Morgante, M., and Michelmore, R.W. 2002. TIR-X and TIR-NBS proteins: Two new families related to disease resistance TIR-NBS-LRR proteins encoded in Arabidopsis and other plant genomes. Plant J. 32: 77-92.

Michelmore, R.W. and Meyers, B.C. 1998. Clusters of resistance genes in plants evolve by divergent selection and a birth-and-death process. Genome Res. 8: 1113-1130.
Nicholas, K.B., Nicholas Jr., H.B., and Deerfield II, D.W. 1997. GeneDoc: Analysis and Visualization of Genetic Variation. EMBNEW NEWS 4: 14

Page, R.D.M. 1996. TREEVIEW: An application to display phylogenetic trees on personal computers. Comput. Appl. Biosci. 12: $357-358$

Pan, Q., Liu, Y.-S., Budai-Hadrian, O., Sela, M., Carmel-Goren, L., Zamir, D., and Fluhr, R. 2000a. Comparative genetics of nucleotide binding site-leucine rich repeat resistance gene homologues in the genomes of two dicotyledons: Tomato and Arabidopsis. Genetics 155: 309-322.

Pan, Q., Wendel, J., and Fluhr, R. 2000b. Divergent evolution of plant NBS-LRR resistance gene homologues in dicot and cereal genomes. J. Mol. Evol. 50: 203-213.

Parniske, M., Hammond-Kosack, K.E., Golstein, C., Thomas, C.M., Jones, D.A., Harrison, K., Wulff, B.B.H., and Jones, J.D.G. 1997. Novel disease resistance specificities result from sequence exchange between tandemly repeated genes at the $C f-4 / 9$ locus of tomato. Cell 91: 821-832.

Perrière, G. and Gouy, M. 1996. WWW-Query: An on-line retrieval system for biological sequence banks. Biochimie 78: 364-369.

Solovyev V.V. 2001. Statistical approaches in Eukaryotic gene prediction. In Handbook of statistical genetics (eds. D. Balding, et al.), pp. 83-127. John Wiley \& Sons, Ltd., New York, NY.

Sun, Q., Collins, N.C., Ayliffe, M., Smith, S.M., Drake, J., Pryor, A., and Hulbert, S.H. 2001. Recombination between paralogues at the $r p 1$ rust resistance locus in maize. Genetics 158: 423-438.

Swofford, D.L. 2002. PAUP. Phylogenetic Analysis Using Parsimony. Version 4. Sinauer Associates, Sunderland, Massachusetts.

Thompson, J.D., Gibson, T.J., Plewniak, F., Jeanmougin, F., and Higgins, D.G. 1997. The ClustalX windows interface: Flexible strategies for multiple sequence alignment aided by quality analysis tools. Nucleic Acids Res. 24: 4876-4882.

Wang, Z.X., Yano, M., Yamanouchi, U., Iwamoto, M., Monna, L., Hayasaka, H., Katayose, Y., and Sasaki, T. 1999. The Pib gene for rice blast resistance belongs to the nucleotide binding and leucine-rich repeat class of plant disease resistance genes. Plant $J$. 19: $55-64$.

Webb, C.A., Richter, T.E., Collins, N.C., Nicolas, M., Trick, H.N., Pryor, T., and Hulbert, S.H. 2002. Genetic and molecular characterization of the maize $r p 3$ rust resistance locus. Genetics 162: 381-394.

Yoshimura, S., Yamanouchi, U., Katayose, Y., Toki, S., Wang, Z.-X., Kono, I., Yano, M., Iwata, N., and Sasaki, T. 1998. Expression of $\mathrm{Xa1}$, a bacterial blight-resistance gene in rice, is induced by bacterial inoculation. Proc. Natl. Acad. Sci. 95: 1663-1668.

Young, N.D. 2000. The genetic architecture of resistance. Curr. Opin. Plant Biol. 3: 285-290.

Yu, J., Hu, S., Wang, J., Wong, K.-S.-G., Li, S., Liu, B., Deng, Y., Dai, L., Zhou, Y., Zhang, X., et al. 2002. A draft sequence of the rice genome (Oryza sativa ssp. indica). Science 296: 79-92.

Zhou, F., Kurth, J., Wei, F., Elliott, C., Vale, G., Yahiaoui, N., Keller, B., Somerville, S., Wise, R., and Schulze-Lefert, P. 2000. Cell-autonomous expression of barley Mla1 confers race-specific resistance to the powdery mildew fungus via a Rar1 independent signaling pathway. Plant Cell 13: 337-350.

\section{WEB SITE REFERENCES}

http://www.ncbi.nlm.nih.gov/; National Center for Biotechnology Information.

http://niblrrs.ucdavis.edu/At_RGenes/; database of Arabidopsis NBS-LRR encoding disease resistance gene homologs.

http://www.rice-research.org/; Monsanto Rice Genome Sequence Database.

http://btn.genomics.org.cn/rice/; Indica rice database from Beijing Institute of Genomics.

Received May 22, 2002; accepted in revised form September 27, 2002. 


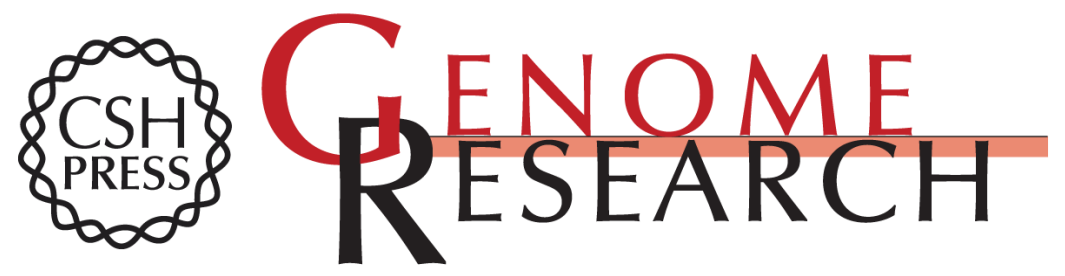

\section{Diversity in Nucleotide Binding Site-Leucine-Rich Repeat Genes in Cereals}

Jianfa Bai, Lourdes A. Pennill, Jianchang Ning, et al.

Genome Res. 2002 12: 1871-1884

Access the most recent version at doi:10.1101/gr.454902

References This article cites 41 articles, 21 of which can be accessed free at:

http://genome.cshlp.org/content/12/12/1871.full.html\#ref-list-1

\section{License}

Email Alerting Receive free email alerts when new articles cite this article - sign up in the box at the Service top right corner of the article or click here.

\section{Affordable, Accurate Sequencing.}

To subscribe to Genome Research go to: https://genome.cshlp.org/subscriptions 